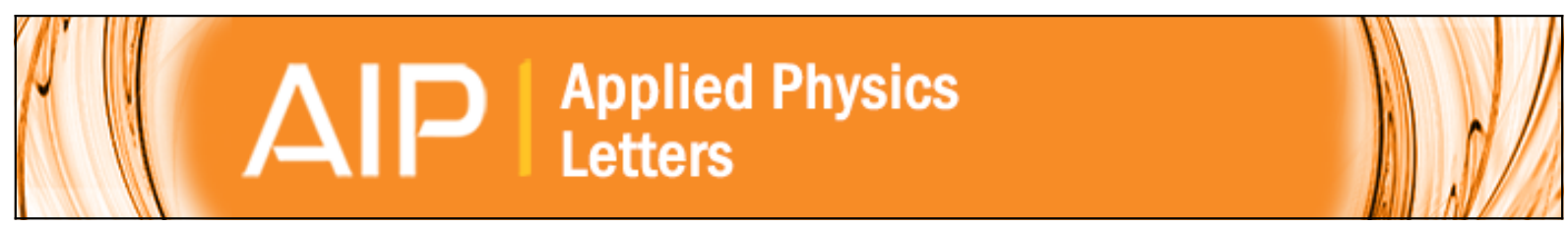

\title{
Magnetic domain wall induced, localized nanowire reversal
}

L. O'Brien, A. Beguivin, A. Fernandez-Pacheco, D. Petit, D. E. Read, and R. P. Cowburn

Citation: Applied Physics Letters 101, 062415 (2012); doi: 10.1063/1.4742746

View online: http://dx.doi.org/10.1063/1.4742746

View Table of Contents: http://scitation.aip.org/content/aip/journal/apl/101/6?ver=pdfcov

Published by the AIP Publishing

$\stackrel{A}{A} \mathbb{P} P$ Re-register for Table of Content Alerts

Create a profile.

Sign up today! 


\title{
Magnetic domain wall induced, localized nanowire reversal
}

\author{
L. O’Brien, ${ }^{1,2, a)}$ A. Beguivin, ${ }^{1}$ A. Fernandez-Pacheco, ${ }^{1}$ D. Petit, ${ }^{1}$ D. E. Read, ${ }^{3}$ \\ and R. P. Cowburn ${ }^{1}$ \\ ${ }^{1}$ Thin Film Magnetism, Cavendish Laboratory, University of Cambridge, Cambridge CB3 OHE, \\ United Kingdom \\ ${ }^{2}$ Department of Chemical Engineering and Materials Science, University of Minnesota, Minneapolis, \\ Minnesota 55455, USA \\ ${ }^{3}$ School of Physics and Astronomy, Cardiff University, Cardiff, CF24 3AA, United Kingdom
}

(Received 14 March 2012; accepted 23 July 2012; published online 9 August 2012)

\begin{abstract}
Considerable difficulties exist in generating appreciable magnetic fields, localized on nanometer length scales for future experiments and technologies. Here we experimentally demonstrate selective reversal of a ferromagnetic nanowire by the stray field from a domain wall. The use of a domain wall as a persistent, mobile source of magnetic field is an alternative to localized Oersted fields and current induced switching, with possible use in future domain wall based data storage schemes and magnetic random access memory applications. (C) 2012 American Institute of Physics. [http://dx.doi.org/10.1063/1.4742746]
\end{abstract}

Many emerging nano-scale experiments and technologies require appreciable magnetic fields, localized on nanometer length scales, including spintronic devices, magnetic random access memories (MRAM), racetrack memories, and quantum information processing devices. ${ }^{1-4}$ Large permanent- or electro-magnets can supply considerable fields but lack spatial confinement. Using the Oersted field from a patterned current-carrying strip offers localized fields, however is incompatible with future device scaling. To this aim, recent research has demonstrated the use of patterned nanoscale ferromagnetic elements as a source of tailorable, highly localized magnetic field. ${ }^{5}$ The stray field from a domain wall (DW), $\boldsymbol{H}_{\boldsymbol{D W}}$, in a planar ferromagnetic nanowire has also been identified as a source of localized magnetic field. ${ }^{6}$ These DWs have the added advantage that they may be propagated (e.g., under the application of a magnetic field) within the nanowires and so may be considered as a highly mobile source of localized field. This mobile field source underpins data writing in proposed DW-based storage devices, ${ }^{3}$ may manipulate magnetic beads for biological sensing, ${ }^{7}$ and has seen theoretical interest in atom trapping. ${ }^{8}$ Furthermore, DWs may be moved using electrical currents ${ }^{9,10}$ allowing an all electrical control of magnetic data writing and manipulation. In this letter we experimentally demonstrate the use of a DW as a mobile source of magnetic field, localized on the nano-meter scale. We show using such a source of field, a magnetic element may be directly and selectively reverseda mechanism potentially highly useful for data writing in magnetic DW data storage and MRAM applications.

In ferromagnetic nanowires (NWs) patterned from magnetically soft material [e.g., permalloy (Py)], reversal of the magnetization is mediated by the nucleation of a DW and occurs when the field exceeds a critical nucleation field threshold (NFT). The magnitude of this field depends on the angle the field makes with the nanowire axes. The nucleation mechanism is well described by the thermally activated reversal of a small magnetic volume, on the order of the char-

\footnotetext{
${ }^{\text {a) }}$ Author to whom correspondence should be addressed. Electronic mail: lao24@cam.ac.uk.
}

acteristic volume of the DW $\left(\sim 10^{-22} \mathrm{~m}^{3}\right.$ in our devices $) .{ }^{11,12}$ This reversal typically occurs at the end of the nanowire and is highly sensitive to the relative angle of the applied field as well as the temperature and experimental wait time for reversal. Once nucleated, it is possible to propagate a DW under a substantially lower amplitude field known as the propagation field, $H_{P}$. For the nanowire geometries discussed, $H_{P} \sim 18$ Oe (average $H_{P}$ obtained over multiple nominally identical individual nanowires) when $\boldsymbol{H}$ is applied parallel to the nanowire long axis.

To experimentally demonstrate the process of DWinduced reversal, Py nanowire devices are fabricated as shown in Figure 1(a). The devices are made by electron beam lithography, thermal evaporation and a lift-off process. Nanowires are $100 \mathrm{~nm}$ wide and $10 \mathrm{~nm}$ thick. Multiple devices are fabricated with varying nanowire separations to probe the spatial extent and effective strength of the DW stray field, $\boldsymbol{H}_{\boldsymbol{D W}}$, used to assist nanowire reversal. In these devices the vertical nanowire acts as a conduit for the DW, hereon termed the conduit. The horizontal nanowire is the nanowire to be switched, hereon termed the element (only the end of the nanowire is shown for clarity). The DW conduit is patterned to be $20 \mu \mathrm{m}$ long in the y-direction; the element is $5 \mu \mathrm{m}$ long in the $x$-direction. The inset figure shows a magnified image of the interaction region for an elementconduit separation of $25 \mathrm{~nm}$. The C-shaped corner allows controllable initialization and propagation of a single DW of known internal structure through the conduit, using a globally applied sequence of magnetic fields, $\boldsymbol{H}_{\boldsymbol{A} p \boldsymbol{p}}$. Figures 1(b) and 1(c) show a schematic of the movement of the DW during the induced-reversal experiment and the applied field sequence, respectively. Initially a large saturating field in the $(-1,-1)$ direction is applied, initializing the device with a DW in the corner of the C-shape and the element in the $-x$ direction [state "i" in Figs. 1(b) and 1(c)]. For the wire cross sectional geometry used in this work, the transverse DW (TDW) is the stable equilibrium DW configuration. ${ }^{13,14}$ Minimization of Zeeman energy due to the applied field and the local potential experienced from the curved conduit section ${ }^{15}$ forces the internal magnetization of the DW to point in the 


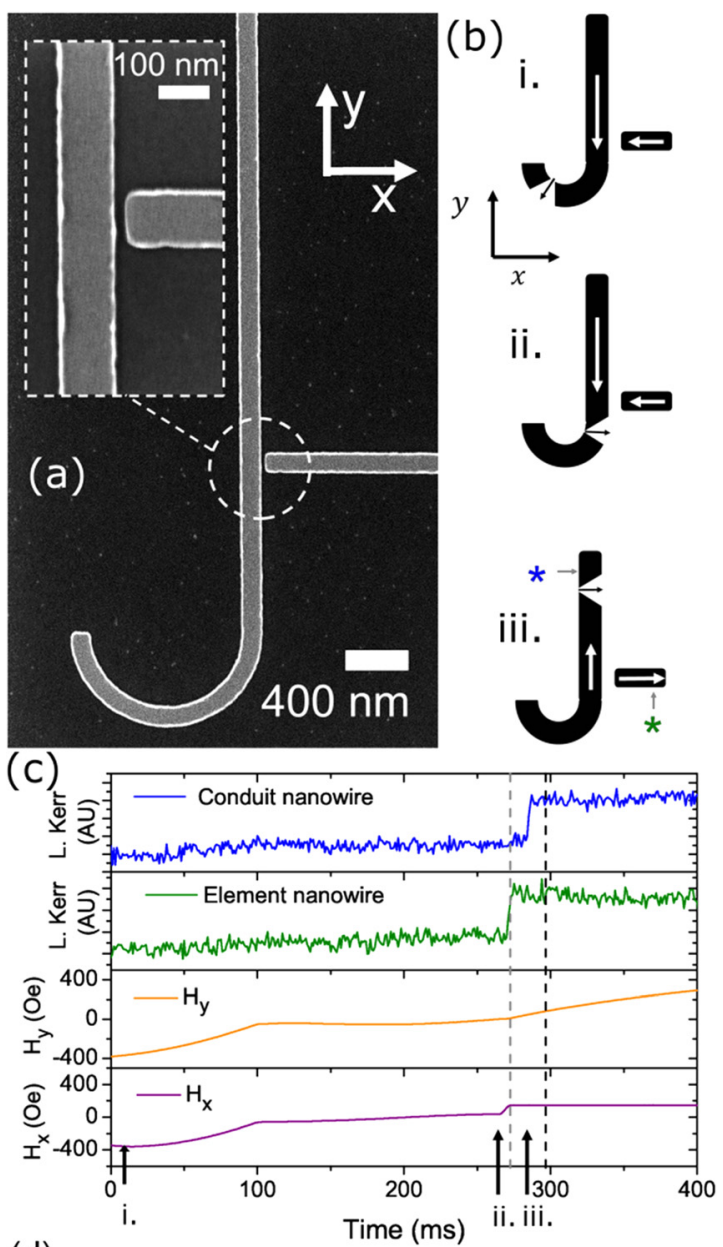

(d)

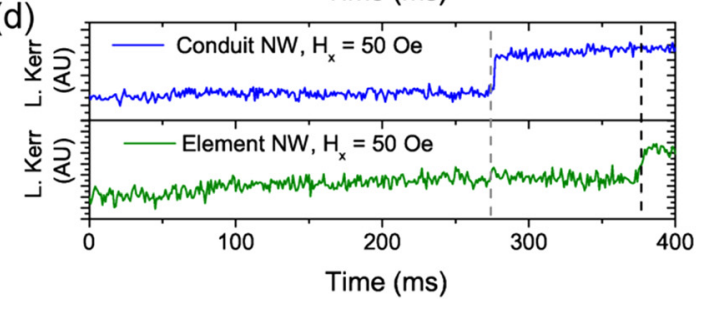

FIG. 1. (a) SEM image of typical tested device. Inset: magnified image of interaction area. (b) Schematic of magnetic evolution under field sequence (for DW-induced reversal). (c) MOKE observed switching of vertical conduit (blue trace) and horizontal element (green trace) under applied global field (DW-induced reversal case). Components of field $H_{y}$ (orange) and $H_{x}$ (purple) are shown for comparison. In this case, the DC $H_{x}$ offset $=150$ Oe. Shown for comparison are $H_{y}=H_{P}$ (grey line) and the relevant NFT. (d) MOKE observed switching for no DW-induced reversal, with DC $H_{x}=50$ Oe: conduit switches at $H_{y} \sim H_{P}$ and element switches at the NFT.

$(-1,-1)$ direction, i.e., a head to head (HH) TDW with widest side along the outer edge of the curved conduit is created. Subsequently, a counter-clockwise rotating field of amplitude 48 Oe moves the DW around the C-shape [state ii]. The DW moves quasi-statically around the corner, with its central magnetization pointing parallel to the applied field direction, thereby avoiding any dynamic changes to its configuration. ${ }^{16}$ Once state ii in Figures 1(b) and 1(c) is reached, a constant DC offset $H_{x}$ (to provide a bias field for element reversal) is applied whilst $H_{y}$ is increased at a constant rate of $\sim 1 \mathrm{Oe} / \mathrm{ms}$. Increasing $H_{y}$ causes the DW to propagate along the conduit, towards the element. The element-corner distance is small ( $\sim 1 \mu \mathrm{m}$ ) to avoid any dynamic changes in the DW configuration, ${ }^{17}$ ensuring the TDW meets the element with its internal magnetization pointing in the $+x$ direction, i.e., $\boldsymbol{H}_{\boldsymbol{D W}}$ experienced by the element will be on average in the $+x$ direction and will assist its reversal. The opposing stray field from the element initially repels the DW when it comes in close proximity, pinning it to a position near the element. On increasing $H_{y}$ further this repulsion is eventually overcome and the DW may continue to propagate through the remaining portion of the conduit. Two possible outcomes can occur as the DW passes the element. First, the combination of the instantaneous amplitude of the applied field, $\boldsymbol{H}_{\boldsymbol{A p p}}$, and DW stray field, $\boldsymbol{H}_{\boldsymbol{D W}}$, are sufficient to reverse the element, i.e., DW-induced reversal of the element occurs. In this case the element is observed to switch for a value of $\boldsymbol{H}_{\boldsymbol{A p p}}$ substantially below the NFT. Alternatively, the combination of the two fields is insufficient to reverse the element and so the DW passes without switching of the element. Switching then occurs at a later time when $\boldsymbol{H}_{\boldsymbol{A p p}}$ has increased such that it exceeds the element NFT. By probing switching of the element and the conduit, as a function of both the DC $H_{x}$ offset and magnitude of $H_{y}$, we may independently probe the effect of $\boldsymbol{H}_{\boldsymbol{D W}}$ on the element switching, and the pinning strength of the DW-element interaction [state iii]. The magnetization of both nanowires is probed experimentally using spatially resolved longitudinal magneto-optical Kerr effect (MOKE) magnetometry at the positions marked with *. Comparable sensitivity to the two orthogonal magnetization directions for the conduit and element is achieved by rotating the relative orientation of the incoming light polarisation, to the sample and magnetic field axes, between measurements of each nanowire. A further experiment is performed to obtain the free switching NFT of the element, in the absence of a DW, but under similar field conditions to the previous DW-induced case. In this case, the $\boldsymbol{H}_{\boldsymbol{A p p}}$ sequence is identical except for a reduction in the amplitude of the initial rotating field to 27 Oe. This reduction is sufficient to prevent the DW from passing around the corner which may be directly observed by measuring the switching field of the conduit at its NFT (instead of at the lower DW-element interaction values found in the DW-present experiments). Subsequent switching of the element is therefore observed without contributions due to the DW.

In Figure 1(c) the switching of the nanowire ends of both the conduit (vertical nanowire; blue trace) and element (horizontal nanowire; green trace) are shown with the components of $\boldsymbol{H}_{\boldsymbol{A p p}}=\left(H_{x}, H_{y}\right)$, averaged over 100 field cycles. For DW-induced reversal experiments the same initializing and rotating field are applied before varying the $H_{x}$ offset to ensure the TDW is always initially present in the vertical conduit. The presence of the TDW can be further verified by measuring switching of the conduit below the conduit NFT. In the MOKE output, a transition from a low to high state indicates switching of the relevant nanowire, and so the passage of a DW under the probe point. If no DW-element interaction occurred, it would be expected that the conduit would first switch for $H_{y}=H_{P} \sim 18$ Oe and the element switch at a later time when $\boldsymbol{H}_{\boldsymbol{A} \boldsymbol{p} \boldsymbol{p}}$ exceeds the NFT. The displayed data traces of Fig. 1(c) are for $H_{x}=150$ Oe during the constant DC offset stage of the field sequence. Indicated by the vertical grey and black lines are the points when $H_{y}=H_{P}$ and the value of $H_{y}$ when nucleation in the element will occur 
(obtained from free switching experiment with no DW present), respectively. From this data we see the vertical conduit switches well above $H_{y}=H_{P}$, i.e., the DW has been pinned. Furthermore, during the experiment the element switches as the DW approaches the element, substantially below the NFT, indicating the vertically propagating DW has directly induced its reversal. That the element switches before the conduit demonstrates that the reversed element has subsequently strongly pinned the DW, as will be discussed later. We may contrast this induced-switching behavior directly with a low-amplitude offset $\left(H_{x}=50 \mathrm{Oe}\right)$ case, as shown in Fig. 1(d). In this case the field sequences follow those shown in Fig. 1(c), however now with a DC offset value of $H_{x}=50$ Oe above $275 \mathrm{~ms}$. Indicated in the plot are $H_{y}=H_{P}$ and the relevant NFT value for the element. In this case, $\boldsymbol{H}_{\boldsymbol{A} p \boldsymbol{p}}+\boldsymbol{H}_{\boldsymbol{D W}}$ is below the NFT when the DW passes the element. Conduit switching now occurs at $H_{y}=H_{P}$ and the element at the NFT value (i.e., at a later time, once the DW has passed), as would be expected for no DW-induced switching contribution. Note, switching of the conduit when $H_{y}$ is slightly above $H_{P}$ is due to the field required to overcome the initially repulsive interaction between the DW and the unswitched element and will also be discussed later.

By varying the $H_{x}$ DC offset in the previous experiments the phase boundary between DW induced reversal and no switching may be experimentally constructed. Figs. 2(a)2(c) show the constructed phase diagram as a function of DC $H_{x}$ bias and maximum applied $H_{y}$ field, for three elementconduit spacings: 25,40 , and $80 \mathrm{~nm}$. As mentioned, the experimental procedure maintains constant $H_{x}$ while increasing $H_{y}$. The plots of Figure 2 should therefore be interpreted by considering increasing $H_{y}$ along lines of constant $H_{x}$. It should be noted that the switching diagram obtained is in the quasi-static limit (field evolution occurs at a rate much slower than DW dynamic timescales). Most importantly, due to this we may only access the effect of $\boldsymbol{H}_{\boldsymbol{D W}}$ at the point in field the DW passes the element. The black points indicate the element switching values for the experiments where the DW is absent from the conduit, i.e., the element NFT, with region 1 (blue) representing when switching may occur purely by $\boldsymbol{H}_{\boldsymbol{A} p \boldsymbol{p}}$. The interaction between DW and element is initially repulsive and so requires a threshold $H_{y}$ to propagate the DW in close proximity. The green line displays the threshold field to overcome this repulsion and switch the conduit, with region 2 showing the regime where the DW does not pass the element. The red data represent the switching field of the element during the DW-induced experiment. For low $H_{x}$ (e.g., $H_{x}<90$ Oe for a $25 \mathrm{~nm}$ separation), when the DW passes the element (i.e., $\boldsymbol{H}_{\boldsymbol{A} p \boldsymbol{p}}$ field values shown by green data) the combination of $\boldsymbol{H}_{\boldsymbol{A p p}}+\boldsymbol{H}_{\boldsymbol{D} W}$ is below the NFT. Due to this the element will remain un-switched during the passage of the DW. The element only subsequently switches at a later time when $\boldsymbol{H}_{\text {App }}$ exceeds the NFT (red, DW-present data follows black, DW-absent points). The yellow region (3) represents maximum applied fields for which $\boldsymbol{H}_{\boldsymbol{A p p}}+\boldsymbol{H}_{\boldsymbol{D W}}$ is insufficient to switch the element at the point the DW passes, and so values for which the element always remains unswitched. It should be reiterated that although the maximum $\boldsymbol{H}_{\boldsymbol{A} p \boldsymbol{p}}+\boldsymbol{H}_{\boldsymbol{D} \boldsymbol{W}}$ may exceed the NFT within region (3), it is the magnitude of $\boldsymbol{H}_{\boldsymbol{A} p \boldsymbol{p}}$ at the point the DW passes
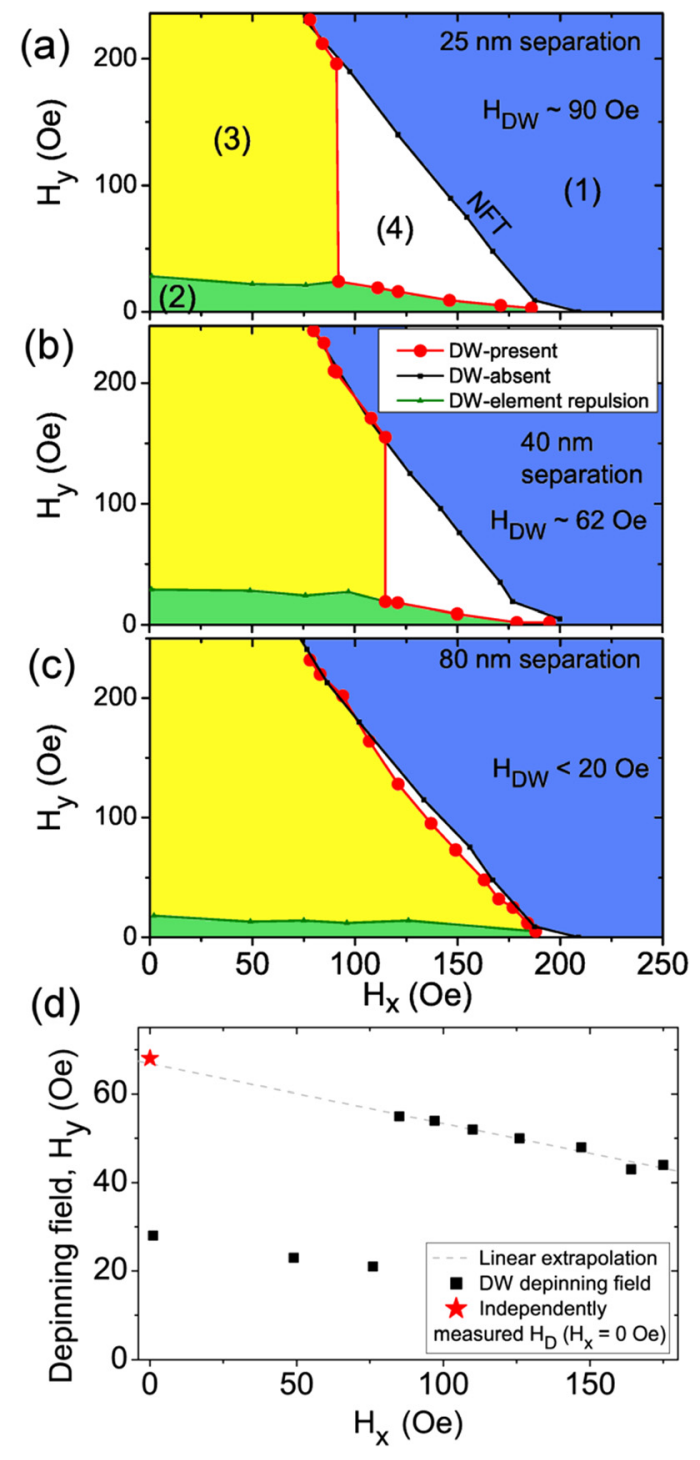

FIG. 2. (a)-(c) Operating area for DW-induced reversal, bound by DWabsent free switching astroid (black data) and DW-induced switching (red data), for conduit-element separations of 25,40 , and $80 \mathrm{~nm}$, respectively. (d) DW depinning field in $y\left(H_{y}\right)$ as a function of applied DC $H_{x}$ bias (black points). Dashed line indicates linear extrapolation from attractive pinning regime. Red star indicates independently measured depinning field at zero bias (see text for details).

the element that will determine switching. For large $H_{x}$ [ $>90$ Oe in Fig. 2(a)], $\boldsymbol{H}_{\boldsymbol{A} p \boldsymbol{p}}+\boldsymbol{H}_{\boldsymbol{D W}}$ exceeds the NFT as the DW passes the element, and so switching of the element now coincides with overcoming the initial DW-element repulsion (red data is seen to drop to much lower values of $H_{y}$, comparable to green points). Region 4 (white) therefore shows the working area for DW-induced reversal in this system. Similar NFTs (DW-absent experiment, black astroid) are observed in all cases, whilst the operating area for DWinduced reversal is found to decrease for increasing interwire spacing: as the spacing increases, the effective $\boldsymbol{H}_{\boldsymbol{D W}}$ experienced by the element-taken as the maximum difference in $H_{x}$ between the DW-present and DW-absent datadecreases $\left(\left|\boldsymbol{H}_{\boldsymbol{D} W}\right|=95 \mathrm{Oe}\right.$ at $25 \mathrm{~nm}$, reducing to $62 \mathrm{Oe}$ at $40 \mathrm{~nm}$ ) thereby requiring a larger $\boldsymbol{H}_{\boldsymbol{A} p \boldsymbol{p}}$ to assist DW-induced reversal. At a distance of $80 \mathrm{~nm}$ the operating area for DWinduced reversal becomes negligibly small, with $\left|\boldsymbol{H}_{\boldsymbol{D W}}\right|$ dropping to $<20$ Oe. 
The effect of the DW stray field upon the switching of an element has been demonstrated. Equally, the stray field of the element will either repel or attract the DW depending on the specific potential landscape experienced. ${ }^{18,19}$ In the case of the experiment conducted, the unswitched element will initially repel the DW. Once switched, however, the interaction will become attractive. The strength of this interaction is indicated by the field required to transmit the DW across the interaction area and switch the vertical conduit, i.e., the DW depinning field. Fig. 2(d) shows the switching field of the conduit as a function of the DC $H_{x}$ bias field for a conduitelement spacing of $25 \mathrm{~nm}$. Two pinning regimes are observed, with a discontinuous jump occurring between the two. This discontinuity agrees well with the minimum $H_{x}$ offset required for DW-induced switching [see Fig. 2(a)] and indicates a transition from a repulsive to attractive interaction after the element has switched. Both interactions have a linear dependence on $H_{x}$ offset; however, there is a considerable difference in pinning strength. The grey dashed line indicates a linear extrapolation of the $H_{x}$ dependence of DW depinning field in the attractive regime. We see this extrapolation agrees extremely well with an independent measurement of the $H_{x}=0$ Oe depinning field value (red star, see Ref. 19 for details of experimental procedure), providing further evidence that it is the attractive interaction observed in this case. Considering the DW and element as rigid objects would result in equivalent pinning strengths for the repulsive and attractive interactions. The results presented would indicate therefore an incompatibility between these results and previous literature work on magnetostatic DW interactions. ${ }^{19-21}$ It would be expected that the magnetic configuration of the unswitched element must distort (towards reversal of the critical volume) in the presence of the DW. We therefore attribute this departure from idealized rigid behavior directly to the distortion of the magnetic element (rather than the DW) whereas, in contrast to the element distortion, simulations indicate the DW remains relatively rigid during the interaction.

Representative images of the nucleation behavior due to DW-induced reversal, obtained from micromagnetic simulations, are shown in Figs. 3(a)-3(d) for the case of a $25 \mathrm{~nm}$ conduit-element separation $(M s=800 \mathrm{kA} / \mathrm{m}, A=13 \mathrm{pJ} / \mathrm{m}$, $\alpha=0.1$, cell size $\left.4 \times 4 \times 4 \mathrm{~nm}^{3}\right){ }^{22}$ For these simulations $2.5 \mu \mathrm{m}$ and $500 \mathrm{~nm}$ long sections of the conduit and element, respectively, are treated to reduce calculation time; only the central interaction region is shown for clarity. The system is initialized as shown in Fig. 3(a), with the element magnetized in the $-x$ direction and the conduit with a HH TDW at the bottom of the figure. The stray fields from the element end and DW oppose one another and so the DW is initially repelled. As in the experimental case, a fixed $H_{x}$ is applied while $H_{y}$ is increased (in $5 \mathrm{Oe}$ steps). We see, in agreement with the experimental case, that if the total field $\boldsymbol{H}_{\boldsymbol{A p p}}+\boldsymbol{H}_{\boldsymbol{D W}}$ is sufficiently large, the element will reverse as the DW comes in close proximity [Fig. 3(c)]. Once the element is reversed, the DW-element interaction becomes attractive and thus pins the DW. On increasing $H_{y}$ further, the pinning of the DW by the element is overcome and the DW propagates along the conduit leaving the conduit in a mono-domain state [Fig. 1(d)]. In the contrary case, where $\boldsymbol{H}_{\boldsymbol{A} p \boldsymbol{p}}+\boldsymbol{H}_{\boldsymbol{D} \boldsymbol{W}}$ is below the

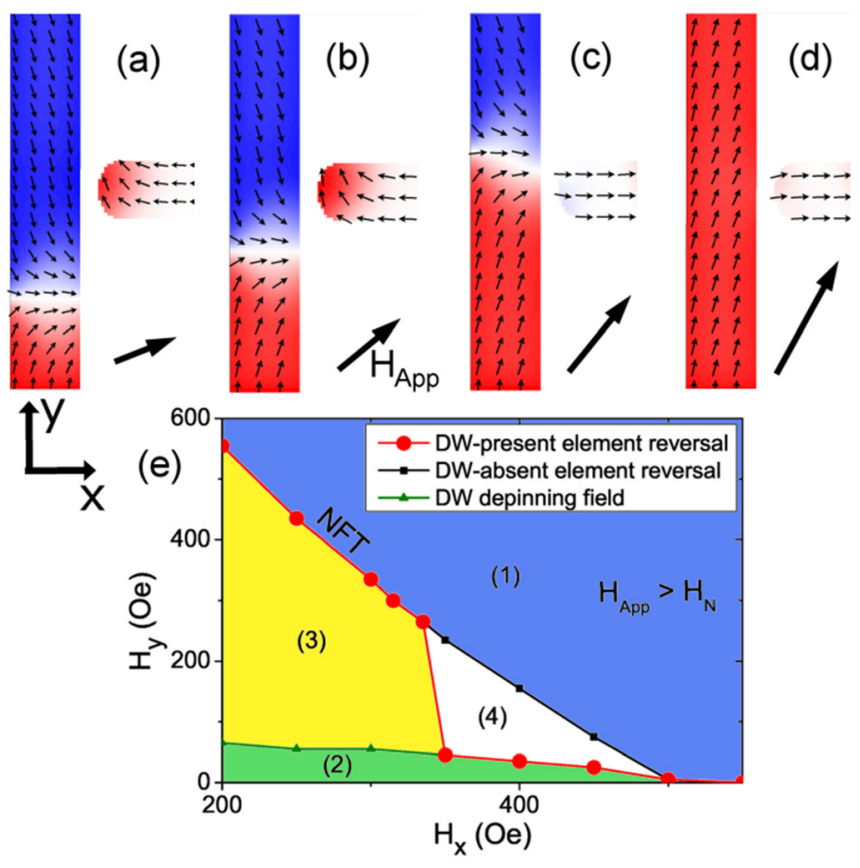

FIG. 3. (a)-(d) Micromagnetic evolution of simulated DW-induced element reversal, for $25 \mathrm{~nm}$ separation between conduit and element. (e) Switching diagram for DW-induced reversal; white area indicates operating region for switching mechanism.

NFT, the DW is observed to propagate through the conduit without switching the element. The element subsequently switches at a later time when $\boldsymbol{H}_{\boldsymbol{A} p \boldsymbol{p}}$ alone has exceeded the NFT. Further simulations were conducted on the same geometry in the absence of the initialized DW, i.e., with a uniformly magnetized conduit in the $-y$ direction. In this case, switching of the element occurs at the NFT and indicates the DWabsent switching fields [black data in Fig. 3(e)].

Fig. 3(e) shows the obtained numerical switching diagram as a function of the maximum $x$ - and $y$-components of $\boldsymbol{H}_{\text {App }}$. Qualitatively identical behavior is seen between micromagnetic simulations and the closest two separations, indicating for the experimental cases investigated that the DW has indeed induced switching in the manner described earlier.

Both experimental results and micromagnetic simulations indicate fields of $\sim 100$ Oe experienced at separations of $25 \mathrm{~nm}$, with simulations indicating $H_{D W}$ increases up to the order of $1 \mathrm{kOe}$ close to the conduit edge. These field strengths are appealing, however they require highly spatially resolved fabrication to be capitalized upon. Despite these demands, the experimental results presented indicate the stray field from a DW may indeed be used effectively to induce reversal, selectively, in an individual nanoscale element.

In conclusion, selective reversal of a ferromagnetic nanowire by the localized stray field from a DW has been experimentally shown. The feasibility of using a DW as a highly mobile and persistent source of magnetic field has been demonstrated, especially as a mechanism for data writing in future data storage applications. The effective switching operating area for DW-induced data writing has been investigated as a function of DW-element separation. Large operating areas and effective magnetic fields are experienced 
at small separations, with both decreasing as DW element separation is increased-in good agreement with numerical simulations. Average fields, experienced over the critical volume to reversal in a nanowire, are estimated to be $95 \mathrm{Oe}$ at $25 \mathrm{~nm}$ separations, with $\sim 1 \mathrm{kOe}$ expected close to the DW conduit edge. This type of DW-induced switching is an alternative to localized Oersted field and current induced switching in magnetic elements, which may be of use to future DW-based data storage schemes and MRAM applications.

The work and results reported in this letter were obtained with research funding from the European Community under the Seventh Framework Programme Contract No. 247368:3SPIN.

${ }^{1}$ S. A. Wolf, D. D. Awschalom, R. A. Buhrman, J. M. Daughton, S. von Molnar, M. L. Roukes, A. Y. Chtchelkanova, and D. M. Treger, Science 294, 1488-1495 (2001).

${ }^{2}$ C. Chappert, A. Fert, and F. N. Van Dau, Nat. Mater. 6, 813-823 (2007).

${ }^{3}$ S. S. P. Parkin, M. Hayashi, and L. Thomas, Science 320, 190-194 (2008).

${ }^{4}$ J. M. Elzerman, R. Hanson, L. H. W. Van Beveren, B. Witkamp, L. M. K. Vandersypen, and L. P. Kouwenhoven, Nature (London) 430, 431-5 (2004).

${ }^{5}$ R. P. G. McNeil, R. J. Schneble, M. Kataoka, C. J. B. Ford, T. Kasama, R. E. Dunin-Borkowski, J. M. Feinberg, R. J. Harrison, C. H. W. Barnes, D. H. Y. Tse, T. Trypiniotis, J. A. C. Bland, D. Anderson, G. A. C. Jones, and M. Pepper, Nano Lett. 10, 1549-53 (2010).

${ }^{6}$ M. Laufenberg, D. Bedau, H. Ehrke, M. Kläui, U. Rudiger, D. Backes, L. J. Heyderman, F. Nolting, C. A. F. Vaz, J. A. C. Bland, T. Kasama, R. E.
Dunin-Borkowski, S. Cherifi, A. Locatelli, and S. Heun, Appl. Phys. Lett. 88, 212510 (2006).

${ }^{7}$ A. Chen, G. Vieira, T. Henighan, M. Howdyshell, J. A. North, A. J. Hauser, F. Y. Yang, M. G. Poirier, C. Jayaprakash, and R. Sooryakumar, Phys. Rev. Lett. 107, 87206 (2011).

${ }^{8}$ D. A. Allwood, T. Schrefl, G. Hrkac, I. G. Hughes, and C. S. Adams, Appl. Phys. Lett. 89, 14102 (2006).

${ }^{9}$ L. Berger, J. Phys. Chem. Solids 35, 947-956 (1974).

${ }^{10}$ N. Vernier, D. A. Allwood, D. Atkinson, M. D. Cooke, and R. P. Cowburn, Europhys. Lett. 65, 526-532 (2004).

${ }^{11}$ W. Wernsdorfer, B. Doudin, D. Mailly, K. Hasselbach, A. Benoit, J. Meier, J. P. Ansermet, and B. Barbara, Phys. Rev. Lett. 77, 1873-1876 (1996).

${ }^{12}$ G. Bertotti, Hysteresis in Magnetism (Academic, San Diego, CA, 1998).

${ }^{13}$ R. D. McMichael and M. J. Donahue, IEEE Trans. Mag. 33, 4167-4169 (1997).

${ }^{14}$ M. Kläui, C. A. F. Vaz, J. A. C. Bland, L. J. Heyderman, F. Nolting, A. Pavlovska, E. Bauer, S. Cherifi, S. Heun, and A. Locatelli, Appl. Phys. Lett. 85, 5637-5639 (2004).

${ }^{15}$ E. R. Lewis, D. Petit, L. Thevenard, A. V. Jausovec, L. O'Brien, D. E. Read, and R. P. Cowburn, Appl. Phys. Lett. 95, 152505 (2009).

${ }^{16}$ N. L. Schryer and L. R. Walker, J. Appl. Phys. 45, 5406-5421 (1974).

${ }^{17}$ E. Lewis, D. Petit, A.-V. Jausovec, L. O'Brien, D. Read, H. Zeng, and R. Cowburn, Phys. Rev. Lett. 102, 057209 (2009).

${ }^{18}$ J. Lili, A. Orlov, G. H. Bernstein, W. Porod, and G. Csaba, in Proceedings of the 9th IEEE Conference on Nanotechnology, 26-30th July 2009, Genoa, Italy, pp. 758-762.

${ }^{19}$ L. O'Brien, D. Petit, E. R. Lewis, R. P. Cowburn, D. E. Read, J. Sampaio, H. T. Zeng, and A.-V. Jausovec, Phys. Rev. Lett. 106, 087204 (2011).

${ }^{20}$ L. O'Brien, D. Petit, H. T. Zeng, E. R. Lewis, J. Sampaio, A. V. Jausovec, D. E. Read, and R. P. Cowburn, Phys. Rev. Lett. 103, 77206 (2009).

${ }^{21}$ S.-M. Ahn, K.-W. Moon, C.-G. Cho, and S.-B. Choe, Nanotechnology 22, 085201 (2011).

${ }^{22}$ M. J. Donahue and D. G. Porter, Interagency Report NISTIR 6376, 1999. 